\title{
Genome-wide Analysis of BPI Transcriptional Targets in Breast Cancer Cell line Hs578T
}

\author{
Yongchun Song ${ }^{1}$, Chengxue Dang ${ }^{1}$, Yebo Fu², Yi Lian 3 , Jenny Hottel ${ }^{3}$, Xuelan Li ${ }^{4}$, Tim McCaffrey $^{3}$ and \\ Sidney W. Fü ${ }^{\circledR}$
}

1. Department of Tumor Surgery, the First Affiliated Hospital, Xi'an Jiaotong University College of Medicine, 277 W. Yanta Road, Xi'an, Shaanxi 710061, China

2. Marriotts Ridge High School, 12100 Woodford Drive, Marriottsville, MD 21104, USA

3. Department of Biochemistry and Molecular Biology, The Catharine Birch McCormick Genomics Center, George Washington University Medical Center, 2300 I Street, Washington DC 20037, USA

4. Department of OB \& GYN, the First Affiliated Hospital, Xi' an Jiaotong University College of Medicine, 277 W. Yanta Road, Xi'an, Shaanxi 710061, China

$\triangle$ Correspondence to: Sidney W. Fu, M.D., Ph.D., Associate Professor, Director, The Genomics Core Facility, Dept. of Biochemistry and Molecular Biology, and of Surgery, Catharine Birch McCormick Genomics Center, The George Washington University Medical Center, 2300 I Street, N.W. Room 536, Washington, DC 20037. Email: sfu@gwu.edu; Tel: 202-994-4767 (Office), 202-994-0691 (Lab); Fax: 202-994-2487

Received: 2008.11.24; Accepted: 2008.12.02; Published: 2008.12.03

Homeobox genes are known to be critically important in tumor development and progression. The BPI (Beta Protein I) gene, an isoform of DLX4, belongs to the Distal-less (DLX) subfamily of homeobox genes and encodes a homeodomain-containing transcription factor. Our studies have shown that the BPI gene was overexpressed in $81 \%$ of primary breast cancer and its expression was closely correlated with the progression of breast cancer. However, the exact role of BPI in breast has yet to be elucidated. Therefore, it is important to explore the potential transcriptional targets of BPI via whole genome-scale screening. In this study, we used the chromatin immunoprecipitation on chip (ChIP-on-chip) and gene expression microarray assays to identify candidate target genes and gene networks, which are directly regulated by BPI in ER negative (ER-) breast cancer cells. After rigorous bioinformatic and statistical analysis for both ChIP-on-chip and expression microarray gene lists, 18 overlapping genes were noted and verified. Those potential target genes are involved in a variety of tumorigenic pathways, which sheds light on the functional mechanisms of BPI in breast cancer development and progression.

Key words: BP1, homeoprotein, ChIP-on-chip, breast cancer, ER

\section{Introduction}

BP1 (GenBank accession no.: AF254115) sequence analysis has revealed that it belongs to the Distal-less (DLX) subfamily of homeobox genes [1], which encodes a homeodomain-containing transcription factor. BP1 is an isoform of DLX4, which has two functionally distinctive splice variants including DLX7 [2]. BP1 and DLX7 are apparent isoforms expressed either as splice variants transcribed from a single gene or driven by different promoters. The sequence similarity extends to nucleotide 565 of BP1 where upstream sequences begin to diverge. Furthermore, using FISH (Fluorescence In Situ Hybridi- zation) and PCR, BP1 was mapped to chromosome 17q21-22, the same region to which DLX7 was localized [1].

BP1 gene expression was absent or very low in normal mammary tissue, but was overexpressed in $81 \%$ of primary breast cancer. In addition, BP1 was expressed in a series of breast cancer cell lines and high levels of BP1 expression in breast cancer cell lines strongly correlates with its tumorigenic potential [3]. BP1 expression was closely correlated with the progression of breast cancer [4]. Furthermore, increased BP1 may impart a survival advantage to 
breast cancer cells, which could be a cause of therapeutic resistance in cancer treatment [5]. Through electrophoretic mobility shift assays (EMSA), site-directed mutagenesis and luciferase reporter assays, it was found that BP1 contributes to the inhibition of apoptosis in MCF7 breast cancer cells challenged with TNFa and that the bcl-2 gene is a direct target of BP1 protein [5].

Therefore, experimental whole genome-scale screening is needed to unveil the potential target genes for BP1 homeoprotein. The recently developed protein-DNA binding assay technique, commonly named ChIP-on-chip, is able to produce comprehen- sive genome-wide location information for specific transcription factors. Identifying the BP1 targets would provide new information of regulatory roles of BP1 in breast cancer and potential novel targets for early detection and intervention. But ChIP-on-chip based evidence for transcription factor binding does not always correlate with the actual transcriptional regulation of the target genes. Thus, we applied this method in conjunction with expression microarray and BP1 motif analysis of the promoter region, to identify candidate target genes that are directly regulated by BP1 in breast cancer cell line Hs578T. The overall scheme is depicted in Figure 1.

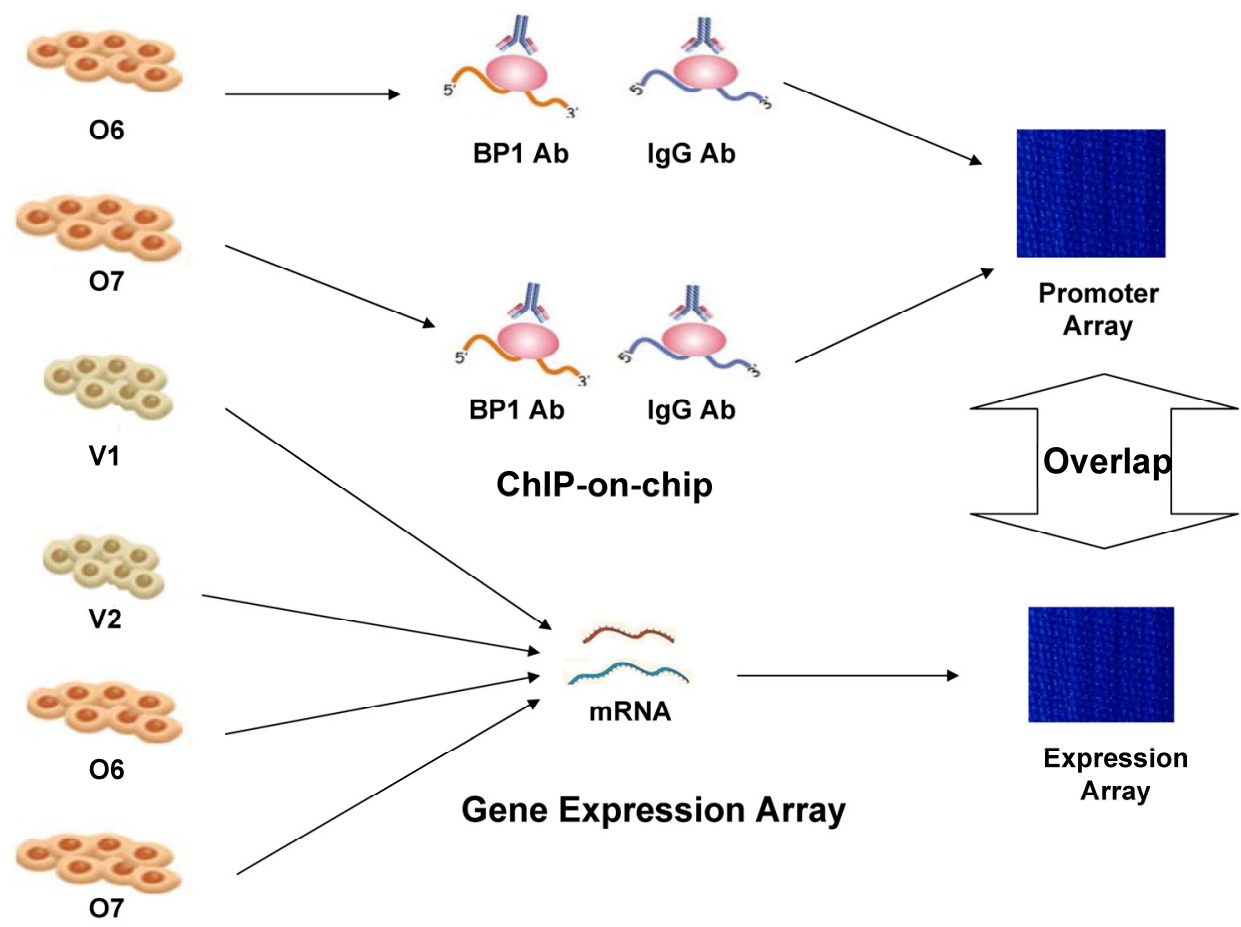

Figure I. Overall experimental design scheme to identify targets of BPI in Hs578T breast cancer cells. ChIP-on-chip was performed on BPI-overexpressing Hs578T cell lines, $\mathrm{O} 6$ and $\mathrm{O7}$. Specific target genes were identified by comparing with lgG-pulled DNA. Affymetrix Human Genome Focus arrays were used to identify differentially expressed genes associated with BPI overexpression using BPI overexpressors, $\mathrm{O} 6$ and $\mathrm{O} 7$ and empty vector controls, $\mathrm{VI}$ and $\mathrm{V} 2$. Overlapping target genes between ChIP-on-chip and expression array analysis were identified for further analysis.

\section{Materials and Methods}

\section{I. Cell Culture / Cell Morphology and MTT assay}

Two Hs578T stable cell lines transfected with pcDNA3.2/BP1 (O6 and O7) were used, along with two controls containing vector pcDNA3.2-DEST alone (V1 and V2). Cells were maintained in DMEM supplemented with $10 \%$ fetal bovine serum, penicillin/streptomycin, 400 $\mu \mathrm{g} / \mathrm{ml} \mathrm{G418} \mathrm{antibiotics,} \mathrm{and}$ $2 \mathrm{mM}$ glutamine. The BP1 expression level of mRNA and protein was confirmed by QPCR and Western Blot assay (Rabbit Polyclonal anti-BP1, NB100-481, NOVUS, Taiwan).

In order to determine cell proliferation rate, the Thiazolyl Blue Tetrazolium Bromide (MTT, Sigma, USA) metabolic assay was employed. Cells were seeded in a 24-well microplate at a concentration of 10,000cells/well. The medium was changed at $12 \mathrm{~h}$. Survival was evaluated by replacing the culture media with $200 \mu \mathrm{l}$ of $0.5 \mathrm{mg} / \mathrm{ml}$ MTT-media solution after $24 \mathrm{~h}, 48 \mathrm{~h}$ and $72 \mathrm{~h}$ incubation. The followed by $4 \mathrm{~h}$ of 
incubation at $37^{\circ} \mathrm{C}$, the MTT reagent was removed before adding $200 \mu \mathrm{l}$ of DMSO. The absorbance was determined at $560 \mathrm{~nm}$ with a microplate reader (Molecular Devices).

Cell images were taken by a Nikon Eclipse TE3000 inverted microscope with $40 \times$ and $100 \times$ magnification (Nikon, Inc., Melville, NY) and MagnaFire software. For the ChIP-on-chip experiment, cells were cultured in a T-150 flask for $24 \mathrm{~h}$ before harvest.

\subsection{Chromatin Immunoprecipitation (ChIP)}

The ChIP-on-chip experiments were performed on two BP1-overexpressing cell lines, O6 and O7. ChIP was carried out using the Upstate kit (New York, USA). The number of cells used was about $5 \times 10^{7}$ for each sample. Protein-DNA cross-links were formed by incubating the cells in $1.42 \%(\mathrm{~V} / \mathrm{V})$ formaldehyde at room temperature for $15 \mathrm{~min}$. Glycine was then added, yielding a final concentration of $0.125 \mathrm{M}$, for $5 \mathrm{~min}$ to stop the cross-linking. Sonication (Sonifier Cell Disrupter 350 model, Branson Ultrasonics Corporation, USA) was performed 11 times, 15 seconds per sonication, to generate $200-500 \mathrm{bp}$ fragments. The lysate was pre-cleared with a protein G/A beads (Calbiochem, USA) slurry for $2 \mathrm{~h}$. A $120 \mu \mathrm{g}$ aliquot of the clear lysate was saved as an "input" control. The remaining lysate was split into 2 eppendorf tubes, and incubated individually with $15 \mu \mathrm{g}$ of specific antibody against BP1 (Rabbit Polyclonal anti-BP1, NB100-481, NOVUS, Taiwan) and $15 \mu \mathrm{g}$ of rabbit IgG (sc-2027, Santa Cruz, USA) at $4^{\circ} \mathrm{C}$ overnight. Next, salmon sperm DNA/protein G/A beads were added to each tube for $2 \mathrm{~h}$ and the beads were pelleted, then eluted. Before phenol-chloroform DNA extraction, the protein-chromatin cross-linking was reversed by proteinase $\mathrm{K}$ for the immunoprecipitated samples and incubated at $65^{\circ} \mathrm{C}$ overnight.

\subsection{Amplification and microarray assay}

The whole-genome amplification (WGA, Sigma) method was used to amplify sufficient amounts of DNA for hybridization to the promoter arrays. The standard protocol for WGA using the GenomePlex® WGA2 kit (Sigma-Aldrich, St. Louis, MO, USA) was adapted with modification of omitting the initial random fragmentation step because ChIP DNA sample is fragmented after sonication. A $10 \mu \mathrm{l} \mathrm{ChIP} \mathrm{DNA}$ samples (IgG and BP1) were amplified. The concentration of the amplified DNA was detected by the nanodrop spectrophotometer. A total of $10.8 \mu \mathrm{g}$ of BP1-pulled DNA and $8.5 \mu \mathrm{g}$ IgG-pulled DNA was amplified.

The microarray chips used for ChIP analysis were GeneChip ${ }^{\circledR}$ Human Promoter 1.0R Array (Af- fymetrix, USA). The fragmentation and labeling was performed with the GeneChip ${ }^{\circledR}$ Terminal Labeling Kit (Affymetrix, USA) according to the manufacturer's recommendations. The final amount of DNA used in hybridization was $7.5 \mu \mathrm{g}$ for each sample. Four GeneChip ${ }^{\circledR}$ Human Promoter 1.0R Array (Affymetrix, USA) chips were used for the analysis. The washing, staining, and scanning were performed as specified by the manufacturer, using the GeneChip ${ }^{\circledR}$ Hybridization Wash and Stain Kit, GeneChip ${ }^{\circledR}$ Hybridization Oven 640, GeneChip ${ }^{\circledR}$ Fluidics Station 450 and GeneChip ${ }^{\circledR}$ Scanner 7G (Affymetrix, USA).

\subsection{Data analysis and verification}

Partek Genomics Suite 6.3 software was used to analyze the data. ChIP-on-chip CEL files were input into the software along with the following parameters: segmentation parameter with three consecutive probes for minimum probe sets, 0.001 for P-value threshold, 1.5 for fold change and 0.5 for signal to noise. One-way ANOVA was used as the statistical method and Affymetrix U133 2.0 Plus Bed file was used to perform a region overlap. A total of 303 significant enrichment binding sites, associated with 259 relevant genes, between BP1 and IgG IP DNA were revealed. For the Human Genome Focused expression array analysis, the One-way ANOVA was used with the parameters of 0.001 for P-value threshold and 2 for fold change, resulting 306 differentially expressed genes. The overlap of the gene lists between the ChIP-on-chip and expression arrays is shown in Figure 4.

To verify the accurate rate for the BP1 binding site that is shown in the ChIP-on-chip list, an independent cell culture and ChIP assay were performed, in the same manner as described above. After precipitation and purification, $25 \mu \mathrm{l} \mathrm{dH} 2 \mathrm{O}$ was used to resolve the ChIP DNA. The $3 \mu$ l of diluted input DNA (100 times dilution) and $3 \mu \mathrm{l}$ of ChIP DNA were used for PCR amplification, in order to verify BP1 binding sites in the IP DNA.

Ten binding sites, whose target genes are relevant to cancer, were selected for verification. RefSeq mapping data was collected from the UCSC Genome Browser's RefFlat table (The UCSC genome browser database: update 2007). As previously described [6], the closest RefSeq 5 end for each ChIP region was identified, regardless of whether the cluster was upstream or downstream of the 5 end. The distance was calculated from the start of each cluster (Tab. 2 and Fig. 6). The Primer3 (v.0.4.0) was used to design the primers that amplifies the binding sites. At the same time, the target gene QPCR primers for mRNA expression analysis were designed to verify the mi- 
croarray results. The PCR results for ChIP DNA and QPCR results of gene expression were shown in Figure 6.

\subsection{Promoter Sequence, Gene Ontology and cascade analysis}

A motif-finding algorithm (MDScan) [7] was utilized to uncover motifs that are highly represented in the set of the promoter sequences. The logo pictured in Figure 3 was generated by WEBLOGO (http://weblogo.berkeley.edu/logo.cgi). Functional categories were assigned using both the Genespring GX 9.0.5 (Agilent, USA) and manual inspection using the Gene Ontology (GO) (www.fatigo.org). In order to predict the relationship between BP1 direct targets and the cell character, Pathway Studio 5.0 (Ariadne, USA) was used to discover the cascades from BP1 direct targets to indirect changed genes.

\section{Results}

\section{I. Cell proliferation and morphology assay}

The BP1 transfected Hs578T cells were established in our lab. The expression of BP1 mRNA was confirmed by QPCR and Western Blot assays (Fig. 2A, 2B). After Hs578T cells were stably transfected by BP1, the cell proliferation ability was detected by the MTT assay. There was significantly more increasing proliferation ability in BP1 transfected Hs578T cells than in vector transfected Hs578T cells (Fig. 2C). During the same time, it was ascertained the cell morphology in BP1 transfected Hs578T cells was changed. The cells became bigger, polygon, and had more antennae in BP1-overexpressing cells. The cells changed from a spindle form to a circular shape (Fig. 2D).
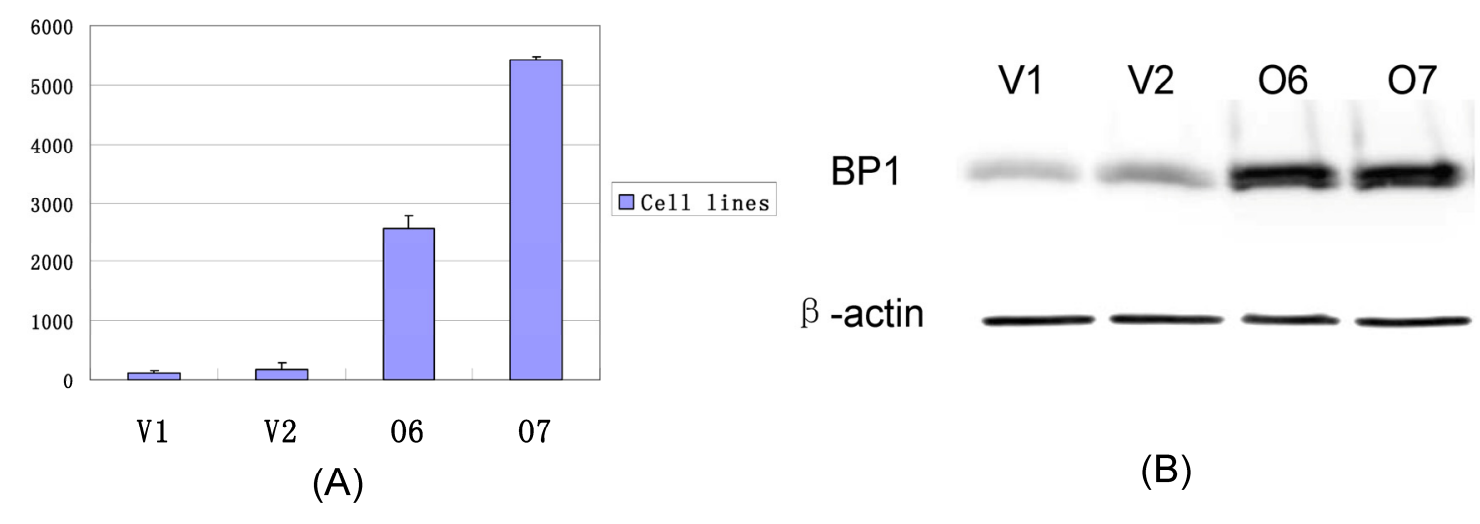

(B)

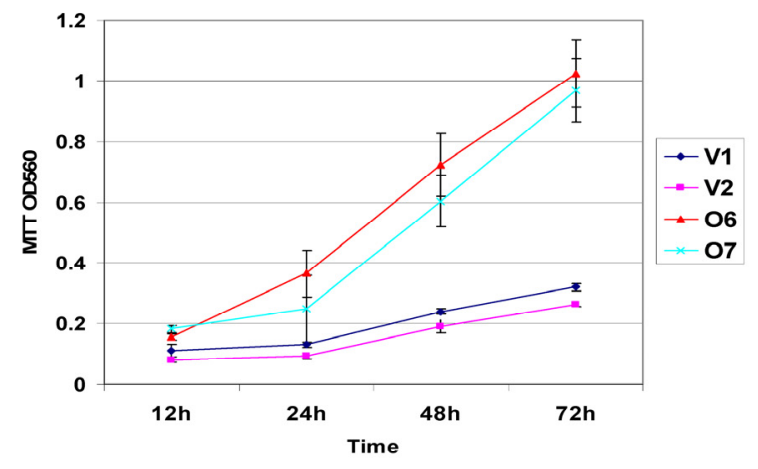

(C)

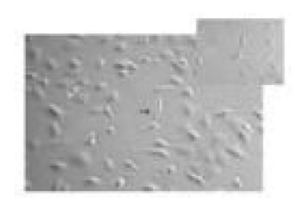

V1

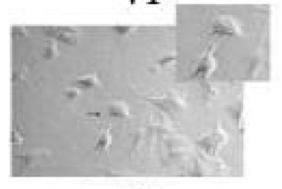

06

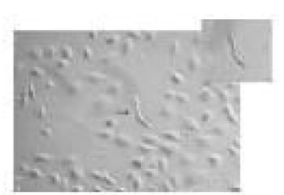

V2

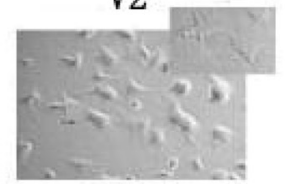

07

(D)

Figure 2. Comparison of the expression, proliferation, and cell morphology between BPI overexpressing cells and controls in Hs578T cells. (A) QPCR analysis of BPI expression. BPI mRNA was expressed more than 100 fold in $\mathrm{O} 6$ and $\mathrm{O} 7$ cells in comparison to the controls, $\mathrm{VI}$ and $\mathrm{V} 2(P<0.0 \mathrm{I})$. (B) Western blot Analysis showing that BPI protein levels are 5 -fold higher in $\mathrm{O} 6$ and $\mathrm{O} 7$ cells than the controls, $\mathrm{VI}$ and $\mathrm{V} 2$. $\beta$-actin was used as an internal control. (C) MTT assay for cell proliferation analysis. At $24 \mathrm{~h}, 48 \mathrm{~h}$ and $72 \mathrm{~h}$, the proliferation rate in $\mathrm{O} 6$ and $\mathrm{O} 7$ cells was significantly higher than that in $\mathrm{VI}$ and $\mathrm{V} 2$ cells $(P<0.0 \mathrm{I})$. The experiments were repeated 3 times. (D) The cell morphology analysis showing the difference between the O6, O7 (Circular) and VI, V2 cells (Spindle). 


\subsection{ChIP-on-chip analysis of BPI binding sites}

To identify targets of BP1 in an unbiased genome-wide manner, the Affymetrix GeneChip ${ }^{\circledR} \mathrm{Hu}-$ man Promoter 1.0R Array, containing the region spanning approximately $7.5 \mathrm{~kb}$ upstream through $2.45 \mathrm{~kb}$ downstream of $5^{\prime}$ transcription start sites of 25,500 human genes, was employed. The $10 \mathrm{~kb}$ tiling array around the transcription start site (TSS) was chosen because more and more evidence has shown the transcription factor bound to the big gap from TSS also modulates the gene expression [8-10].

In order to accurately locate the DNA binding sites of BP1, the experiment was performed with two
BP1-overexpressing cell lines. A total of 153 promoters $(P<0.001)$, bound by BP1 in Hs578T cells (Supplementary Table S1, which is published as supporting information on the web site), were identified. Binding by BP1 to a subset of targets was confirmed using conventional ChIP assays and PCR for 10 binding sites. The rate of false positives was $<20 \%$ when previously established threshold criteria were used (Fig. 6). The distance from binding start site to 5 transcription start site was derived from the UCSC Genome Browser. The primers used in 10 binding sites are available online (Table 1 ).

Table I. Primers used for binding site identification and expression verification.

\begin{tabular}{|c|c|c|}
\hline Gene Symbol & Binding Site Identification & Expression Verification \\
\hline \multirow[t]{2}{*}{ VEGFA } & GAAGGTGCCCAGGTGAGAG & СТACСТCCACCATGCCAAGT \\
\hline & САAATCTCTCCAGAGCСТCAA & GCAGTAGCTGCGCTGATAGA \\
\hline \multirow[t]{2}{*}{ STAT1 } & TCTCACAAGAGGCTGGAGGT & CTTGTTGGGGCACAAGGT \\
\hline & GGCTCTGCTCGCCTACTCT & CCATGGGAAAACTGTCATCA \\
\hline \multirow[t]{2}{*}{ EIF4E } & TACAGTCGGGGAAGGAAGTG & TCGATCTAAGATGGCGACTG \\
\hline & ACAGAACTGACCAGAAACCATC & CTGGGTTAGCAACCTCCTGA \\
\hline \multirow[t]{2}{*}{ MEIS1 } & TCTTTTCCCAGGCTGTCTTC & CATGGGAGGTTTCGTAATGG \\
\hline & TGATATCAATCATAGGTGTATAGCTCA & CCTTGCTTTGCGATTGGTTA \\
\hline \multirow[t]{2}{*}{ MSR1 } & AGGGTGATGAGATGACAGAAA & TGCAGTTCTCATCCCTCTCA \\
\hline & TCAAGGTTAATTTGTTGGGAAC & CCTTTTCCCGTGAGACTTTG \\
\hline \multirow[t]{2}{*}{ ITGA9 } & GAGCTCAAAAGTGCССТCTC & GAAGACCGCGATGATGAGT \\
\hline & TGAGGGAGGAAAAAGAAGCA & GGATGTGGTCGGCTTCATAG \\
\hline \multirow[t]{2}{*}{ HPSE2 } & GGCAAGTTGGATCGGAGTTA & GGCCCGGATTACTATCTCAA \\
\hline & CGCAGGAGATTTAAGGAGGA & GGAGCTCCAGCATAACATCAG \\
\hline \multirow[t]{2}{*}{ IL2RA } & CATGTGGTGCCAGCGTATAG & CCACAGAAATGCAAAGTCCA \\
\hline & GACGCTCACAGTCTCCTCAA & CCCCACCACGAAATGATAAA \\
\hline \multirow[t]{2}{*}{ LAPTM5 } & ACCAGCTGTGTGTCTTGCAG & CTGATCTCCAGCTTCCTGCT \\
\hline & TCCTGTCСТTTAAACССТTTCTC & CCATGATTTGCAGGGACAG \\
\hline \multirow[t]{2}{*}{ MMP27 } & TTCCAGTCAGGCCAGATTTC & CCCTGGGTGGAGAAAATACA \\
\hline & GCTCTGGGTTTTGGAGAATG & TTGCTCCACACTTCTAAACСТC \\
\hline
\end{tabular}

The results of the genome location experiment of BP1 were further analyzed using a motif-finding algorithm (MDscan), which examines the ChIP-on-chip selected sequences and searches for DNA sequence motifs representing the protein-DNA interaction sites [7]. The consensus sequence derived from the most frequent motifs found in the BP1-bound promoters corresponds to a perfect BP1 response element (G/C)CTGCATG(T/C), which is visually represented in Figure 3. This sequence will be used to predict the binding site of BP1 protein in the genome.

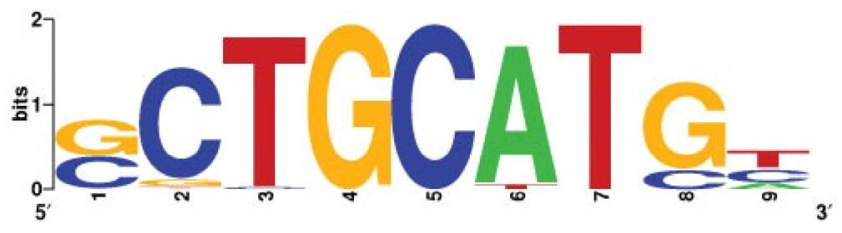

Figure 3. Genome-wide location analysis of direct BPI transcriptional targets in Hs578T breast cancer cells. Motif-finding algorithms identify the consensus BPI sequence $(\mathrm{G} / \mathrm{C}) \mathrm{CTGCATG}(\mathrm{T} / \mathrm{C})$ as the most common transcription factor-binding motif, which presents in $60 \%$ of the promoters used for the analysis. 


\subsection{Gene expression microarray analysis}

The gene expression microarray assay was performed to generate a differentially expressed gene list to identify the potential targets in common with that from the ChIP-on-chip assays. Levels of mRNA expression for each target were compared between vector transfected Hs578T cells and BP1 transfected Hs578T cells. The data from the Human Genome Focused arrays were analyzed using the Partek with strict thresholds $(\mathrm{p}<0.01$, Foldchange $>2)$. The result- ing 306 differentially expressed genes were intensely analyzed by Gene Ontology. Because our experiment was performed in cancer cells, the focus was on cellular physiological processes (GO: 50875). According to the biology function classification, 42 of 306 genes were associated with apoptosis, 32 were related to cell cycle progress, and 17 were involved in cell migration. It was shown that the mechanism of BP1 enhancing proliferation maybe because BP1 effects the apoptotic factors [2].

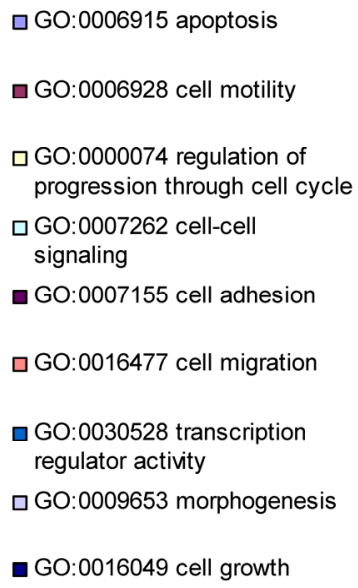

Figure 4. Pie chart representing major biological functions and processes associated with BPI overexpression. The resulting 306 differentially expressed genes were intensely analyzed by Gene Ontology. In cellular physiological process classification, the major part was apoptosis followed by cell motility, cell cycle, cell-cell signaling, cell adhesion, cell migration, transcription regulator activity, morphogenesis, and cell growth.

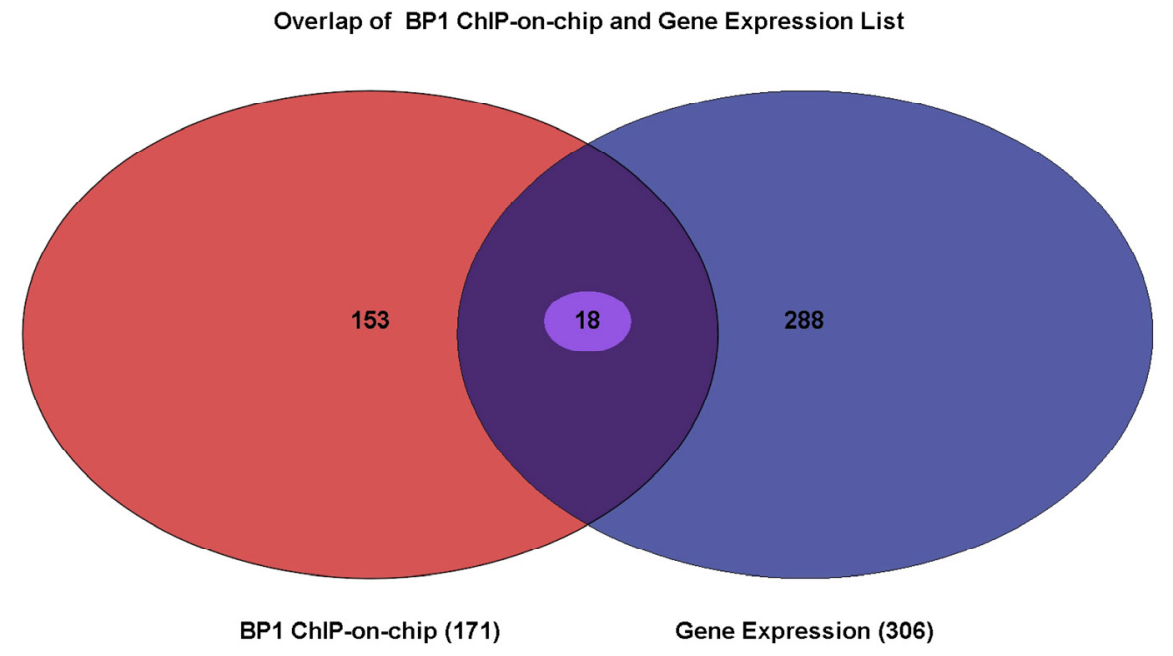

Figure 5. Venn Diagram showing the overlapping genes between gene lists from BPI ChIPon-chip and expression microarray. Of I7I genes from the BPI ChIP-on-chip data and the 306 genes that were differentially expressed, there were 18 of them overlapping. The intersecting region represents 18 genes whose promoter region may be bound by $\mathrm{BPI}$ and that are deregulated when BPI is overexpressed. 


\subsection{Identification of direct gene targets that are expressed significantly different when BPI is overexpressed.}

To elucidate which gene BP1 binds to and deregulates, the Venn Diagram analysis through Partek was performed. There were 18 overlapping genes. The list for these 18 genes is shown in Table 2. The distance in the table represents the number of bases that use 5 transcription start site of the gene minus the binding start site. If this number is positive, the binding site is in front of the TSS, possibly in the promoter or enhancer region. If it is negative, the binding site maybe in the intron or exon of the target gene.

Table 2. Overlapping genes between BPI ChIP-on-chip data and the microarray results

\begin{tabular}{|c|c|c|c|c|}
\hline Gene Symbol & GenBank ID & $\begin{array}{l}\text { Binding Site } \\
\text { Distance (bp) }\end{array}$ & Fold Change & Gene Function \\
\hline ADAM21 & NM_003813 & 4280 & -2.12012 & proteolysis \\
\hline CASQ1 & NM_001231 & 3641 & -1.77249 & mitochondrion \\
\hline CBR1 & NM_001757 & 2651 & -2.15584 & carbonyl reductase (NADPH) activity \\
\hline CCL4 & NM_002984 & -13867 & -1.78511 & cell adhesion \\
\hline CSF2RB & NM_000395 & -690 & -2.24506 & signal transduction \\
\hline HPSE2 & NM_021828 & -1075 & -1.53345 & hydrolase activity \\
\hline HTR1B & NM_000863 & 2552 & -2.0201 & signal transduction \\
\hline IL2RA & NM_000417 & 6214 & -2.42635 & positive regulation of apoptosis \\
\hline ITGA9 & NM_002207 & -2198 & 3.26587 & Cell adhesion \\
\hline LAPTM5 & NM_006762 & 3836 & -2.24236 & integral to plasma membrane \\
\hline LGALS13 & NM_013268 & -248 & -1.93361 & lipid degradation \\
\hline LSM7 & NM_016199 & -3962 & 2.32186 & mRNA metabolism \\
\hline MMP27 & NM_022122 & 600 & -1.53542 & proteinaceous extracellular matrix \\
\hline OR1A1 & NM_014565 & 504 & -2.81835 & signal transduction \\
\hline PTPRE & NM_006504 & -137517 & -1.79625 & protein amino acid dephosphorylation \\
\hline SELE & NM_000450 & 1369 & -1.61012 & cell adhesion \\
\hline TAC3 & NM_013251 & 31955 & -1.68822 & neuropeptide signaling pathway \\
\hline VEGFA & NM_001025370 & -3313 & 2.55763 & angiogenesis, differentiation \\
\hline
\end{tabular}

* Distance was calculated between the positions of 5 transcription start site and potential binding site, which were obtained from UCSC Genome Browser. Positive numbers signify the binding sites are in front of the TSS, and negative ones indicate they are located in the exon or intron regions.

\subsection{Verification by ChIP and QPCR}

Since the amount of genomic DNA obtained from the ChIP is limited, amplification is essential to generate sufficient DNA for microarray hybridization. The method previously used was random primer amplification, which is recommended by Affymetrix

(http://www.affymetrix.com/products/arrays/speci fic/human_promoter.affx). The method chosen in this study was WGA (Whole Genome Amplification). There are many labs now using this method and reporting very consistent data with low bias effects, and large quantities of DNA produced [11].

To verify the potential binding sites from the ChIP-on-chip data, PCR was performed using prim- ers flanking the region of interest; QPCR was also used for the verification of the microarray expression data. Ten sets of primers were designed for both ChIP-on-chip and expression analysis using the Primer3 (v.0.4.0). The UCSC Genome Browser analysis for binding sites prediction, PCR binding site verification and the corresponding QPCR expression analysis is shown in Figure 6. In those 10 conventional ChIP assays, 9 of them were consistent to the ChIP-on-chip assay. The data for ITGA9 shows the same level of DNA recruitment in both BP1 and IgG immunoprecipitated samples, which suggest there might be a weak binding. The QPCR showed all of them had the same gene expression trend as the gene expression array. 


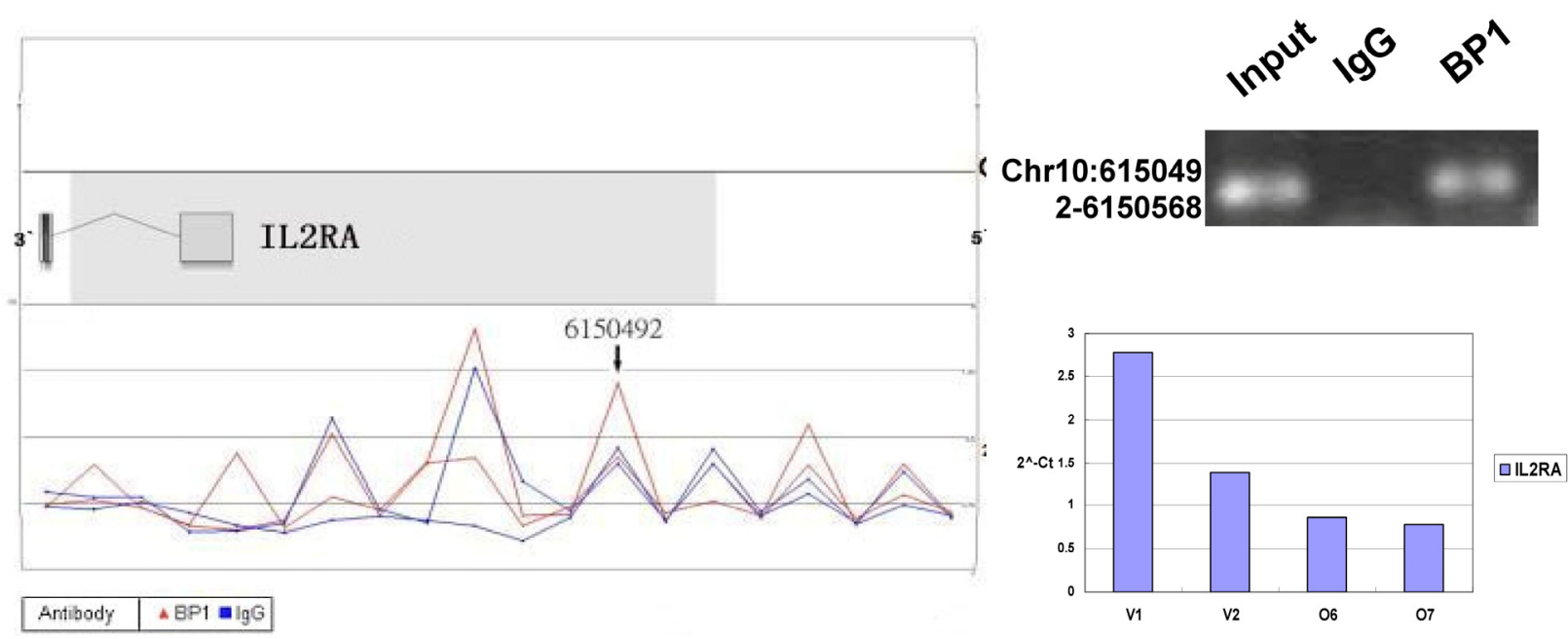

IL2RA
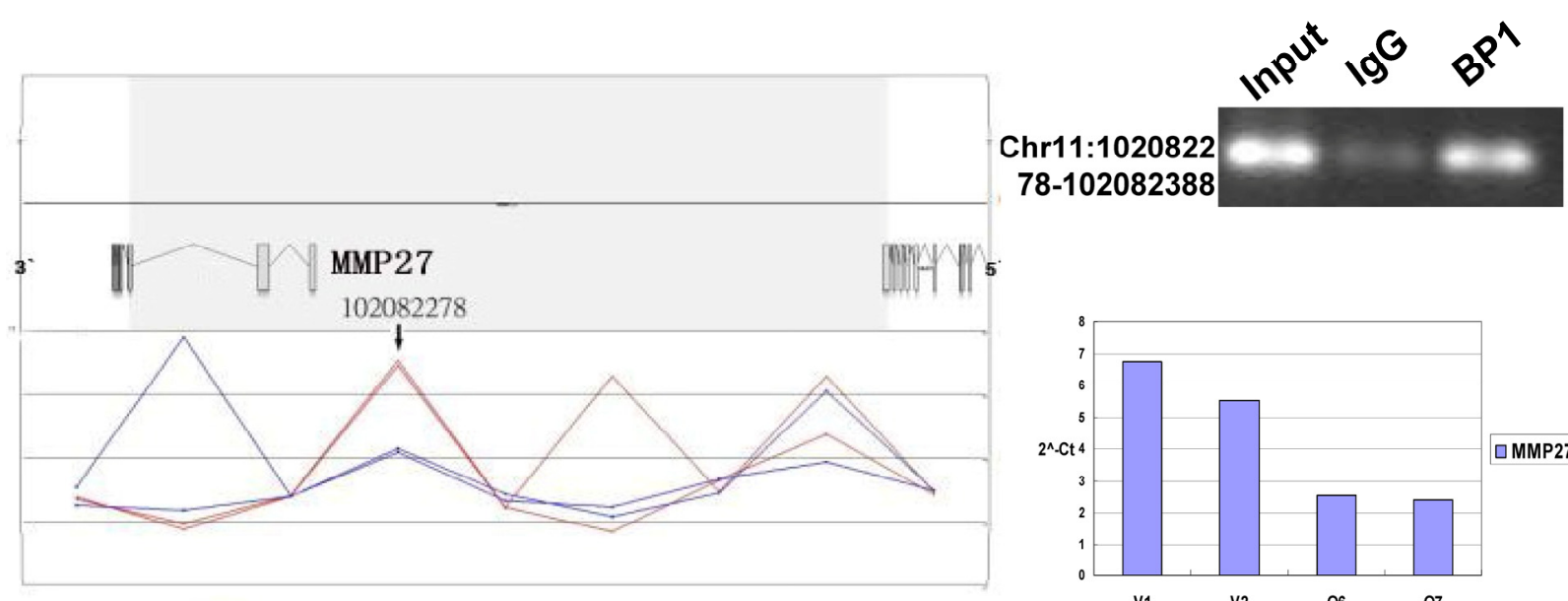

\begin{tabular}{|l|l|}
\hline Antibody & แ BP1 $\mathbf{\text { IgG }}$ \\
\hline
\end{tabular}

MMP27
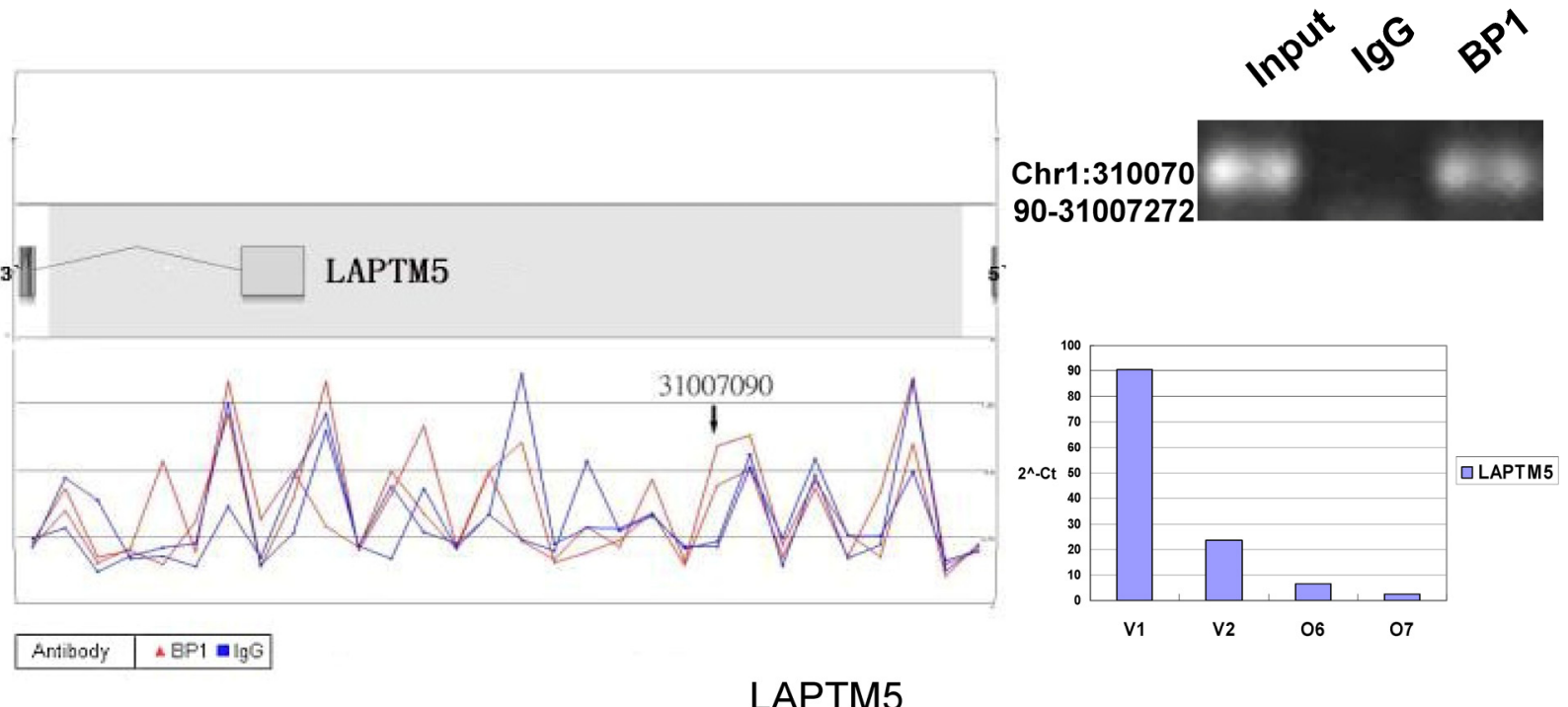

LAPTM5

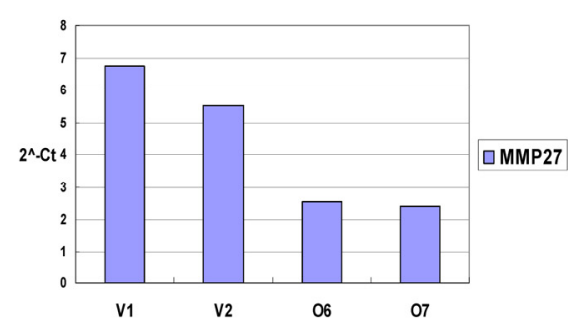




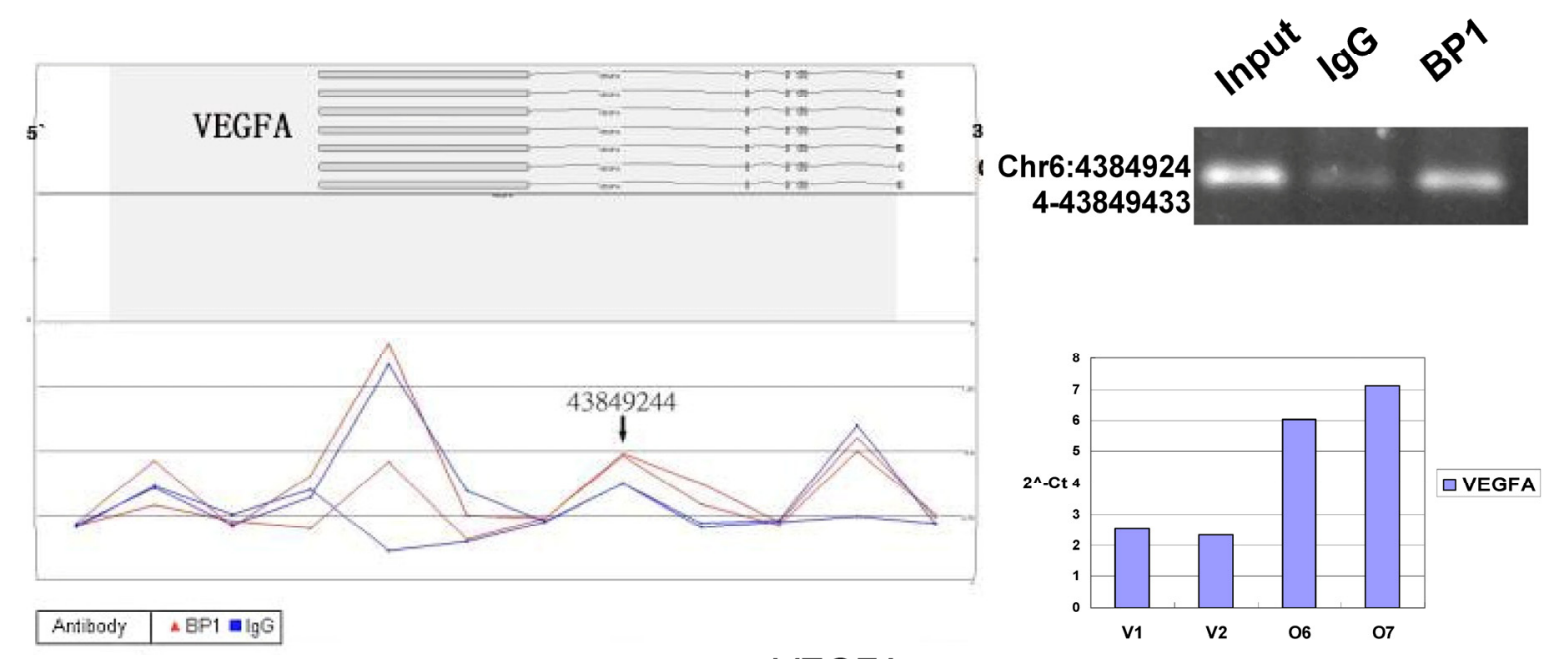

VEGFA

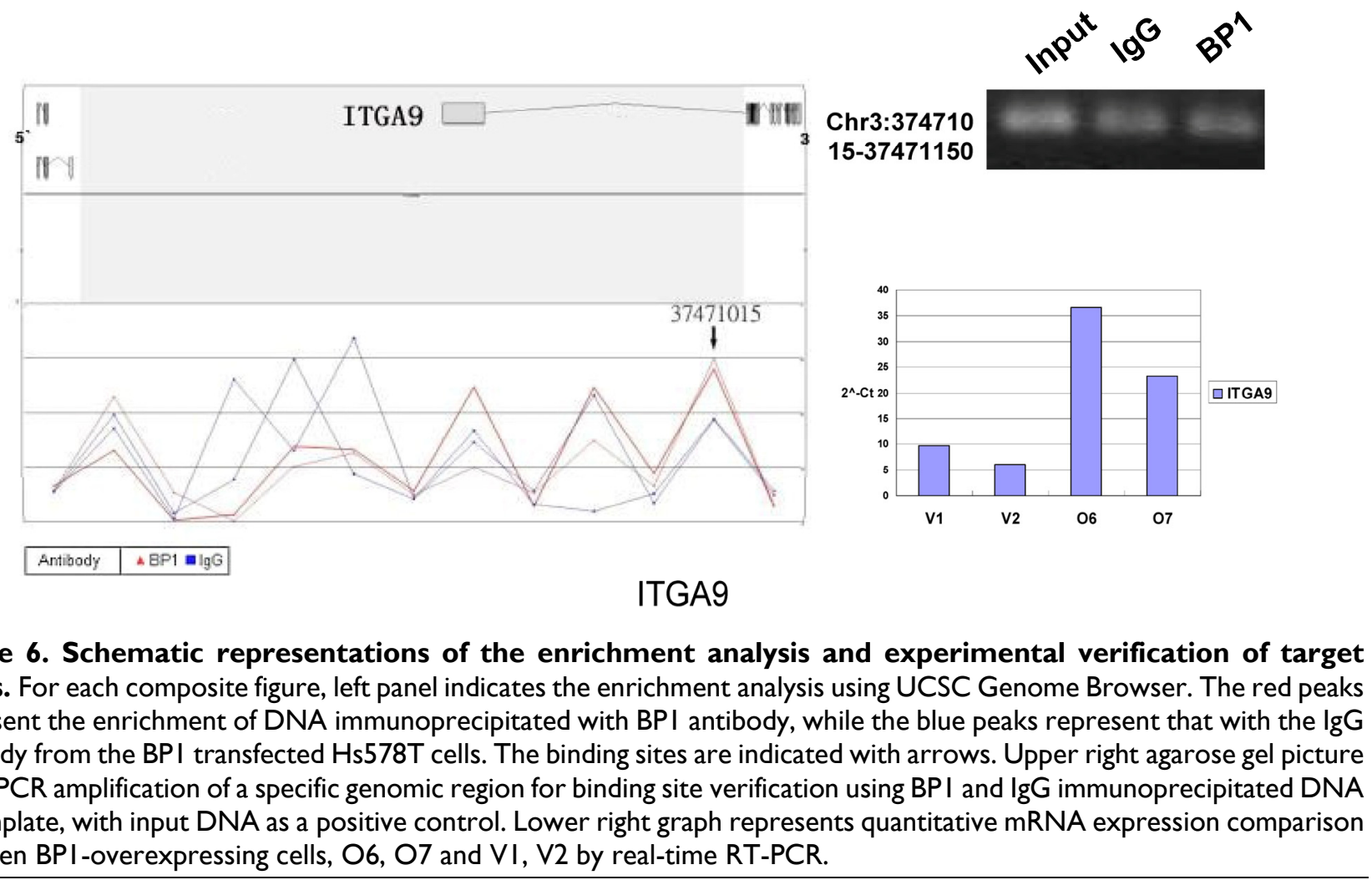

\subsection{Pathway analysis}

Pathway Studio 5.0 was used to extensively analyze the data from both promoter and expression arrays. It is found that multiple differentially expressed genes from the expression array analysis are the targets of the genes that are directly regulated by $\mathrm{BP} 1$. Logically, it is believed that the reason BP1 in- duces cell proliferation and enhances tumor metastasis is the result of direct or indirect downstream regulation. The cascade between VEGFA up-regulation and cell proliferation was shown in Figure 7. 


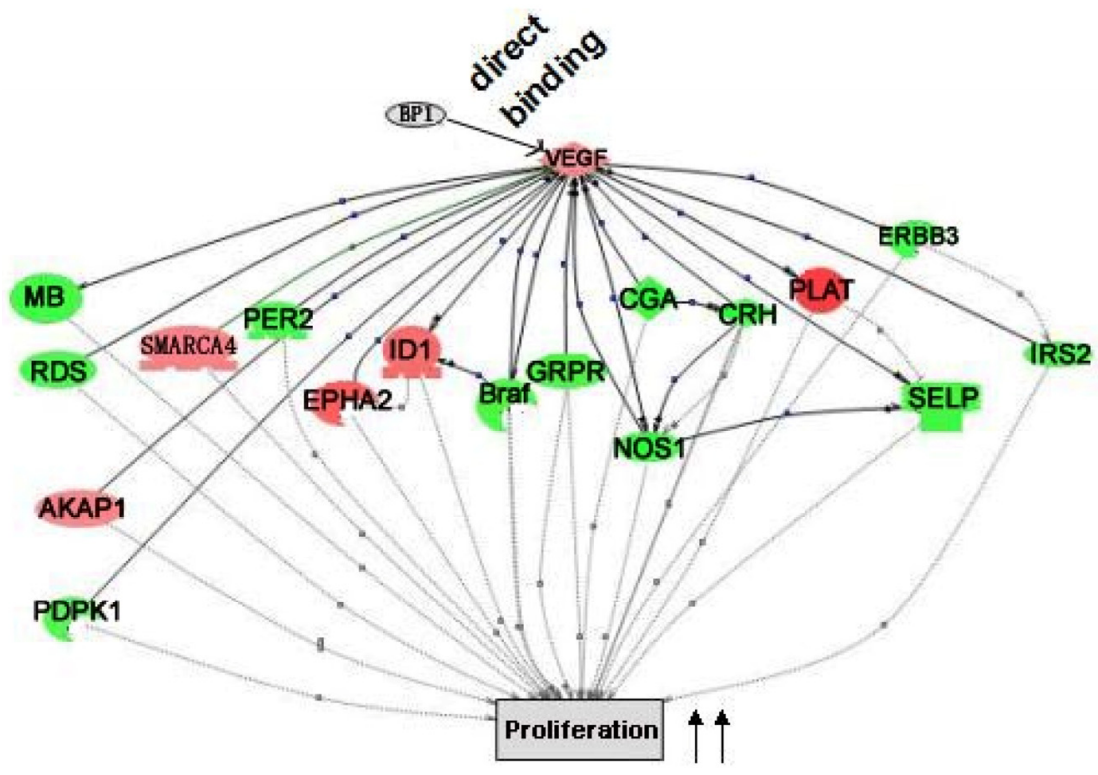

Figure 7. Cascade between VEGFA, a direct target of BPI, and its downstream target genes in cell proliferation. VEGFA functional cascades were analyzed by the Pathway Studio 5.0. Genes in the network, red for upregulated and green for down-regulated ones, are the targets of VEGFA, which collectively promote cell proliferation.

\section{Discussion}

Breast cancer is one of the most common malignancies affecting women and is expected to be diagnosed in approximately 184,450 cases in the United States in 2008, and about 40,480 women will die from the disease this year alone [12]. Homeobox genes are known to be critically important in the normal development of organisms from Drosophila to humans [13]; and cancer is a consequence of normal development gone awry. It is logical to hypothesize that aberrant expression of these genes may contribute to cancer [14]. Actually, a lot of evidence has shown homeobox genes that are upregulated in cancer normally show expression patterns that are restricted to undifferentiated or proliferative cells [15]. Conversely, homeobox genes that are downregulated in cancer are normally expressed in differentiated tissues. Homeobox genes can be re-expressed in the tumorigenic cells that are derivatives of embryonic cells in which the particular homeobox gene is normally expressed during development [16].

The deregulated expression of homeobox genes has been described in many solid tumors and derivative cell lines. Among these, HOX, HSIX1, PAX have been shown to promote cellular transformation in breast cancer[17-19]. The human homologue HOXA1 has been detected in breast cancer lesions and adjacent tissues [20]. HOXA1 mRNA expression is also modulated by retinoic acid, a common differentiation agent in MCF7 cells [21]. In addition, overexpression of murine Hoxb7 in SkBr3 breast adenocarcinoma cells caused increased proliferation and decreased growth factor dependency [22].

It was previously shown that the BP1 gene was overexpressed in $81 \%$ of primary breast cancer [3] and was closely correlated with the progression of breast cancer [4]. Furthermore, in order to elucidate the mechanism of BP1 function, it was discovered that the bcl-2 gene is a direct target of BP1 protein in MCF-7 cells, and contribute to the inhibition of apoptosis challenged with TNFa [5]. Our previous studies have shown that overexpression of BP1 presents a more aggressive phenotype in breast cancer cells (unpublished). The reasons for choosing the Hs578T cell line as a model are because: 1) Hs578T cells are estragon-receptor(ER) negative. There are no effective treatments for ER- breast cancer and often the prognosis is poor; 2) Hs578T cell line expressed low or barely detectable BP1 [3]. Therefore, it is important to identify potential downstream targets of BP1 to elucidate its biological functions in breast tumorigenesis and progression. Homeobox genes usually work as vital genes in embryogenesis and development [23]. Due to the fact that homeobox genes encode transcription factors which typically switch on cascades of other genes, and that BP1 belongs to the DLX family of homeobox genes, it is believed that BP1 regulates its target genes via direct binding to its cor- 
responding promoter or enhancer. Hence, the ChIP-on-chip strategy was used to identify the potential binding targets for $\mathrm{BP} 1$ homeoprotein.

The antibody is one of the most important factors for the ChIP-on-chip experiment. Western blot was performed on the ChIPed lysate to verify the specificity of BP1 immunoprecipitation. The result of the Western Blot showed BP1 protein was bound by this particular BP1 antibody not only in vivo and but also following formaldehyde treatment. Therefore, we believe this antibody is suitable for the identification of BP1 target genes.

The accuracy rate of microarray data depends on the threshold chosen for the analysis. Although the $p$ value in our analysis was 0.001 , we can still identify 10 potential binding sites, of which 9 of them were verified by a conventional ChIP assay. Therefore, the false positives rate was $10 \%$ using our threshold criteria (see Materials and Methods). False positives can occur for a number of reasons in ChIP-on-chip experiments. Therefore, it is recommended to perform independent ChIP experiments when ChIP-on-chip results are intended for further analysis.

New target genes, within the functional categories, that are involved in cell proliferation and other important functions of cancer were identified. Given the known property of BP1 to stimulate cell proliferation and change cell morphology, it was surprising that only a few key genes were obtained in our location analysis, for example, VEGFA and HPSE2.

Binding of BP1 to the promoter regions of target genes is a vital step of BP1 function as a transcription factor. However, the binding of BP1 in the promoter region may not necessarily function as a transcriptional activator or repressor depending on the molecular context. By comparing the expression microarray and binding sites target gene lists, 18 overlapping genes were obtained. Interestingly, most of these genes were down-regulated by BP1, showing that BP1 works as an inhibitor in most situations. Due to our luciferase assay system not working well with down-regulated genes, they could be a subject of future study using a more amenable reading system.

Many predictions can be generated from the established VEGFA cascade network. For example, BP1 directly up-regulates VEGFA expression according to ChIP and QPCR assay (Fig. 6). As reported previously, VEGF enhances the expression of ID [24], which can promote cell proliferation[24-27]. VEGF and ID1 both induce EPHA2 expression[27, 28], which also promote cell proliferation[29, 30]. This demonstrates that $\mathrm{BP} 1$ is an upstream regulator in the VEGF pathway.
Despite the stringency of the integrated analysis of ChIP-on-chip and microarray data, we found a number of novel BP1 target genes, some of which are vital to cell proliferation and tumorigenesis. Of note, further analysis of the BP1 regulatory networks in tumorigenesis is warranted. Take note that the gene expression microarrays used only represent about 9,000 genes, therefore whole genome arrays for gene expression and higher density tiling arrays for ChIP-on-chip assays would permit comprehensive target gene screening. Furthermore, BP1, as a homeoprotein, it may not affect the gene expression for some targets by itself alone, which is probably why the expression of ITGA9 was not changed even though there is clear evidence that BP1 binds to its promoter.

The results of these studies provide new information of regulatory roles of BP1 in breast cancer and potential new targets for early detection and intervention, which call for in depth research using patient samples for biomarker and new drug discovery.

\section{Abbreviations}

DLX: Distal-less gene; BP1: Beta Protein 1; ER: Estrogen Receptor; ChIP: Chromatin Immunoprecipitation; EMSA: Electrophoretic Mobility Shift Assay; QPCR: Quantitative Polymerase Chain Reaction; MTT: 3-(4,5-dimethylthiazol-2-yl)-2,5-diphenyl tetrazolium bromide; WGA: Genome-wide Amplification; TSS: Transcription Start Site.

\section{Acknowledgements}

This work was supported by NIH grant CA102928 (SWF) and the McCormick Genomics Grant (SWF). The authors would like to thank Dr. Allan L. Goldstein for his support and the technical assistance from the Genomics Core Facility at GW.

\section{Conflict of Interest}

The authors have declared that no conflict of interest exists.

\section{References}

1. Chase MB, Fu S, Haga SB, Davenport G, Stevenson H, Do K ,et al. BP1, a homeodomain-containing isoform of DLX4, represses the beta-globin gene. Mol Cell Biol. 2002; 22:2505-2514

2. Fu S, Stevenson H, Strovel JW, Haga SB, Stamberg J, Do K, et al. Distinct functions of two isoforms of a homeobox gene, BP1 and DLX7, in the regulation of the beta-globin gene. Gene. 2001; 278:131-139

3. Fu SW, Schwartz A, Stevenson H, Pinzone JJ, Davenport GJ, Orenstein JM ,et al. Correlation of expression of BP1, a homeobox gene, with estrogen receptor status in breast cancer. Breast Cancer Res. 2003; 5:R82-87

4. Man YG, Fu SW, Schwartz A, Pinzone JJ, Simmens SJ, Berg PE. Expression of BP1, a novel homeobox gene, correlates with 
breast cancer progression and invasion. Breast Cancer Res Treat. 2005; 90:241-247

5. Stevenson HS, Fu SW, Pinzone JJ, Rheey J, Simmens SJ, Berg PE. BP1 transcriptionally activates bcl-2 and inhibits TNFalpha-induced cell death in MCF7 breast cancer cells. Breast Cancer Res. 2007; 9:R60

6. Gao H, Falt S, Sandelin A, Gustafsson JA, Dahlman-Wright K. Genome-wide identification of estrogen receptor alpha-binding sites in mouse liver. Mol Endocrinol. 2008; 22:10-22

7. Liu XS, Brutlag DL, Liu JS. An algorithm for finding protein-DNA binding sites with applications to chromatin-immunoprecipitation microarray experiments. Nat. Biotechnol. 2002; 20:835-839

8. Melnikova L, Biryukova I, Kan T, Georgiev P. Long-distance interactions between regulatory elements are suppressed at the end of a terminally deficient chromosome in Drosophila melanogaster. Chromosoma. 2008; 117:41-50

9. Sipos L, Gyurkovics H. Long-distance interactions between enhancers and promoters. FEBS J. 2005; 272:3253-3259

10. Spirito F, Bossi L. Long-distance effect of downstream transcription on activity of the supercoiling-sensitive leu-500 promoter in a topA mutant of Salmonella typhimurium. J Bacterio. 1996; 178:7129-7137

11. O'Geen H, Nicolet CM, Blahnik K, Green R, Farnham PJ. Comparison of sample preparation methods for ChIP-chip assays. BioTechniques. 2006; 41:577-580.

12. [Internet] American Cancer Society. http://www.cancer.org/ downloads/STT/2008CAFFfinalsecured.pdf.

13. Krumlauf R. Hox genes in vertebrate development. Cell. 1994; 78:191-201.

14. Abate-Shen C. Deregulated homeobox gene expression in cancer: cause or consequence? Nat Rev Cancer. 2002; 2:777-785

15. Nunes FD, de Almeida FC, Tucci R, de Sousa SC. Homeobox genes: a molecular link between development and cancer. Pesqui Odontol Bras. 2003; 17:94-98

16. van Agthoven T, van Agthoven TL, Portengen H, Foekens JA, Dorssers LC. Ectopic expression of epidermal growth factor receptors induces hormone independence in ZR-75-1 human breast cancer cells. Cancer Res. 1992; 52:5082-5088

17. Ford HL, Kabingu EN, Bump EA, Mutter GL, Pardee AB. Abrogation of the G2 cell cycle checkpoint associated with overexpression of HSIX1: a possible mechanism of breast carcinogenesis. Proc Natl Acad Sci U S A. 1998; 95:12608-12613.

18. Peverali FA, D'Esposito M, Acampora D, Bunone G, Negri M, Faiella $\mathrm{A}$, et al. Expression of HOX homeogenes in human neuroblastoma cell culture lines. Differentiation. 1990; 45:61-69

19. Silberstein GB, Van Horn K, Hrabeta-Robinson E, Compton J. Estrogen-triggered delays in mammary gland gene expression during the estrous cycle: evidence for a novel timing system. Journal Endocrinol. 2006; 190:225-239

20. Chariot A, Castronovo V. Detection of HOXA1 expression in human breast cancer. Biochem Biophys Res Commun. 1996; 222:292-297

21. Chariot A, Moreau L, Senterre G, Sobel ME, Castronovo V. Retinoic acid induces three newly cloned HOXA1 transcripts in MCF7 breast cancer cells. Biochem Biophys Res Commun. 1995; 215:713-720

22. Care A, Silvani A, Meccia E, Mattia G, Peschle C, Colombo MP. Transduction of the SkBr3 breast carcinoma cell line with the HOXB7 gene induces bFGF expression, increases cell proliferation and reduces growth factor dependence. Oncogene. 1998; 16:3285-3289.

23. Boncinelli E. Homeobox genes in development. Adv Neurol. 1999; 79:81-94

24. Sakurai D, Tsuchiya N, Yamaguchi A, Okaji Y, Tsuno NH, Kobata $\mathrm{T}$, et al. Crucial role of inhibitor of DNA binding/differentiation in the vascular endothelial growth fac- tor-induced activation and angiogenic processes of human endothelial cells. J Immunol. 2004; 173:5801-5809

25. Chaudhary J, Sadler-Riggleman I, Ague JM, Skinner MK. The helix-loop-helix inhibitor of differentiation (ID) proteins induce post-mitotic terminally differentiated Sertoli cells to re-enter the cell cycle and proliferate. Biol Reprod. 2005; 72:1205-1217

26. Harper EG, Alvares SM, Carter WG. Wounding activates p38 map kinase and activation transcription factor 3 in leading keratinocytes. J Cell Sci. 2005; 118:3471-3485

27. Straume O, Akslen LA. Strong expression of ID1 protein is associated with decreased survival, increased expression of ephrin-A1/EPHA2, and reduced thrombospondin-1 in malignant melanoma. Br J Cancer. 2005; 93:933-938

28. Jiang P, Li JH, Luo GQ, Liu J. [Effect of EphA2 protein on the expression of VEGF and MMP9 proteins in HCT116 cells]. Zhong Nan Da Xue Xue Bao Yi Xue Ban. 2007; 32:679-683

29. Liu F, Park PJ, Lai W, Maher E, Chakravarti A, Durso L ,et al. A genome-wide screen reveals functional gene clusters in the cancer genome and identifies EphA2 as a mitogen in glioblastoma. Cancer Res. 2006; 66:10815-10823

30. Nasreen N, Mohammed KA, Antony VB. Silencing the receptor EphA2 suppresses the growth and haptotaxis of malignant mesothelioma cells. Cancer. 2006; 107:2425-2435 